\title{
Die Entwicklung der Flachsbearbeitungsgeräte im Baltikum in der zweiten Hälfte des 19. und zu Beginn des 20. Jh.
}

Neben der Getreidewirtschaft war der Flachsbau ein wichtiger landwirtschaftlicher Produktionszweig. Im 19. Jh. verfügte Russland über 66 bis 82 Prozent sämtlicher Leinanbauflächen der Erde und war im europäischen Flachshandel führend. Drei Fünftel des in den Ländern Europas angekauften Flachses stamimten aus Russland, und zwar hauptsächlich aus den westrussischen Gouvernements Pskow (Pleskau), Nowgorod, Smolensk, Witebsk, Minsk, Grodno sowie aus den baltischen Gouvernements Livland, Kurland, Wilna und Kowno ${ }^{\mathbf{1}}$. Der nächstgelegene Umschlagplatz für den in Westrussland und im Baltikum erzeugten Flachs war der Rigaer Hafen, in dem um die Mitte des 19. Jh. die Hälfte des gesamten russischen Exportleins verschifft wurde. 45 bis 50 Prozent davon stammten aus den westrussischen Gouvernements, 30 bis 40 Prozent aus Iitauen, 7 bis 8 Prozent aus Kurland und 8 bis 10 Prozent aus Livland ${ }^{2}$.

Folgende Übersicht zeigt die von den baltischen und westrussischen Gouvernements im Flachsbau erzielten Produktionsziffern ${ }^{3}$ :

1 Der Flachsbau war auch in den Gouvernements Twer, Petersburg, Wjatka, Wladimir, Jaroslaw und Kostroma entwickelt. Die in diesen Gebieten erzeugte Leinfaser wurde jedoch vorwiegend in der örtlichen Textilindustrie verwendet.

${ }^{2}$ Die Prozentsātze wurden nach den von J. Zutis (Latvija klaušu saiemniecības sairšanas periodā un Kauguru nemieri. Riga, 1953, S. 24) gemachten Angaben errechnet.

${ }^{3}$ История Латвийской ССР. I. Рига, 1952, S. 553. 


\begin{tabular}{|c|c|c|c|c|}
\hline 1) & Gouvernement & Pskow & 2.600 .000 & Pud \\
\hline 2) & 4 & Smolensk & 1.366 .000 & $"$ \\
\hline 3) & $\#$ & Livland & 750.000 & ") \\
\hline 4) & 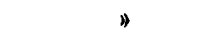 & Kowno & 500.000 & ") \\
\hline 5) & $"$ & Witebsk & 350.000 & 1) \\
\hline 6) & * & Minsk & 250.000 & 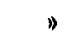 \\
\hline 7) & $n$ & Kurland & 140.000 & 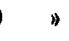 \\
\hline 8) & 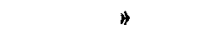 & Wilna & 140.000 & " \\
\hline 9) & $\Rightarrow$ & Grodno & 80.000 & ") \\
\hline
\end{tabular}

Wie aus der Tabelle hervorgeht, standen die baltischen Gouvernements Livland und Kowno unmittelbar nach den bedeutendsten flachsproduzierenden Gebieten, den Gouvernements Pskow und Smolensk, an dritter und vierter Stelle.

Die auf den Export ihrer Erzeugnisse orientierten flachsbauenden Gouvernements Russlands waren in einem gewissen Grade auf die Produktion bestimmter Güter spezialisiert. Die westrussischen und baltischen Gouvernements lieferten hauptsächlich Faserflachs sowie zur Aussaat bestimmte Leinsamen. Die in Riga zur Ausfuhr gelangende Leinsaat erfreute sich in Europa eines guten Rufes und war besonders wegen ihres geringen Gehalts an anderen Samen geschätzt. Die Rigaer Kaufleute erhielten Leinsaat aus Litauen (40 bis 80 Prozent), Kurland (20 bis 40 Prozent) und Livland (20 Prozent). Qualitätsmässig waren die aus Litauen und Kurland stammenden Leinsamen den anderen überlegen ${ }^{1}$. Sehr anerkennend äusserten sich darüber die Organisatoren der Warschauer Industrieausstellung von 1858: "Die Hauptlocalitäten für den russischen Lein als Öl- und Gespinstpflanze sind Kurland, das Gouvernement Pskow, Livland, Witebsk, Kowno und Wilna; hier gewinnt man vorzugsweise jene vortreffliche Leinsaat, welche in Deutschland, Belgien und Frankreich als sogenannter Frühlein ein überaus geschätztes Saatgut abgiebt.'²

${ }^{1}$ И. Грасмане, Значение торгового пути реки Даугавы в лоставке әкспортных товаров в Ригу в конце XУІІІ и первой половине XIX века. - Экономические связи Прибалтики с Россией. Рига, 1968, S. 179 .

2 D. Kerndt, Über Hanf, Flachs und daraus erzeugte Gegenstände auf der Warschauer Industrie-Ausstellung. - Kurländische landwirtschaftliche Mitteilungen (im folgenden - KLM), 1858, Nr. 1. 
Aus Mittelrussland erhielt Riga Faserflachs, Werg und Ölsamen, letztere wurden auch aus den südrussischen Gouvernements geliefert. ${ }^{1}$

Den Anbau von Flachs als einer wichtigen und arbeitsintensiven Kultur betrieben hauptsächlich die Bauernwirtschaften, teils auf eigenem Boden, teils auf gepachtetem Gutsland. Der Flachsbau wurde durch den Umstand gefördert, dass diese Kultur für die Bauern eine ergiebige Einnahmequelle war.

Nachstehende Tabelle zeigt den Anteil der Flachsanbauflächen an der Gesamtfläche der landwirtschaftlichen Kulturen in den soer Jahren des 19. Jh. und zu Beginn des 20. Jh.: ${ }^{2}$

\begin{tabular}{lcccc}
\hline $\begin{array}{l}\text { Gouver- } \\
\text { nement }\end{array}$ & $\begin{array}{c}\text { Flachsanbauflächen } \\
\text { (in 1000 Desjatinen) }\end{array}$ & $\begin{array}{r}\text { Anteil des Flachses an den } \\
\text { Sommersaatkulturen (\%) }\end{array}$ \\
\hline & $1883-1887$ & $1905-1909$ & $1883-1887$ & $1905-1909$ \\
\hline & & & & \\
Pskow & 81 & 102 & 25,3 & 27,8 \\
Smolensk & 36 & 99 & 15,8 & 19,8 \\
Livland & 66 & 73 & 18,4 & 16,7 \\
Kowno & 51 & 45 & 11,3 & 8,4 \\
Wilna & 21 & 19 & 11,2 & 3,8 \\
Witebsk & 36 & 50 & 10,7 & 10,9 \\
Kurland & 12 & 15 & 5,2 & 5,7 \\
Estland & 2 & 5 & 1,8 & 3,3
\end{tabular}

Wie aus der Tabelle zu ersehen ist, stand Livland am Ende des 19. Jh. als Leinanbaugebiet nach Pskow an zweiter Stelle; es folgten Kowno und Wilna. Livland behielt seine Bedentung als eines der wichtigsten Flachsbaugebiete auch zu Beginn des 20. Jahrhunderts. Besonders entwickelt war der Flachsbau in den livländischen Kreisen Walk, Wenden (Cēsis), Wolmar (Valmiera) und Fellin (Wiljandi), wo die Leinanbauflächen 15-20 Prozent der Gesamtfläche der landwirtschaitlichen

${ }^{1}$ Die zum Export bestimmten Leinsamen wurden bis zum 31. Mai des nächsten Jahres als Saatgut, nach Ablauf dieser Frist aber nur noch als Ölsamen verkauft.

${ }^{2}$ Сельскохозяйственные и статистические сведения по материалам, полученным от хозяев. Вып. У. СПб., 1892, S. 334, 504, 514. 
Kulturen ausmachten. ${ }^{1}$ Im Gouvernement Kurland wurde Flachs hauptsächlich in den Kreisen Bausk, Mitau (Jelgava) und Friedrichstadt (Jaunjelgava) angebaut; in diesen drei Kreisen waren 74 Prozent sämtlicher Leinanbauflächen des Gouvernements konzentriert. ${ }^{2}$ Das bedeutendste Flachsbaugebiet Litauens war Rakiškieni.

Entsprechend der Bedeutung, die der Anbau von Flachs als Marktware für die baltischen Bauernwirtschaften hatte, wurden in den Historisch-ethnographischen Atlas des Baltikums acht Kartogramme aufgenommen, die die Verbreitung der folgenden Flachsbaugeräte und -verfahren illustrieren:

1. Geräte zum Abschlagen der Samenkapseln;

2. Erstbearbeitung der Flachsfaser (eventuelles Rösten, Trocknen auf offenem Felde in Bündeln oder ungebündelt);

3. 'Trocknen der Samenköpfchen;

4. Dörren der Flachsfaser vor dem Brechen (in der Korndarre, der Sauna oder einer Dörrgrube);

5. Geräte zum Brechen des Flachses;

6. Schwingverfahren;

7. Geräte zum Dreschen der Samenkapseln;

8. Arten der Flachsbrechmaschinen.

Es handelt sich hierbei ausschliesslich um Geräte, die am Ende des 19. und zu Beginn des 20. Jh. benutzt wurden. Die Nachrichten über Flachsaufbereitungsgeräte aus dem Ende pes 18. und dem Beginn des 19. Jh. sind äusserst dürftig und für die Erarbeitung von Kartogrammen nicht ausreichend. Die Autoren des Atlasses - A. Vischnewskaite (Litauen), V. Kalits (Estland) und I. Leinasare (Lettland) - stützten sich in ihrer Arbeit auf die Erfahrungen von Frau Toini-Inkeri Kaukonen (Bearbeitung von Flachs und Hanf in Finnland. Helsinki, 1946) sowie auf die Arbeiten über bäuerliche Flachsaufberei-

${ }^{2}$ Fr. Jung-Stilling, Ein Beitrag zur livländischen Agrarstatistik. Riga, 1881, S. 20.

2 Экономические очерки Курляндской губернии. Митава, 1897, S. $37-38$. 
tungsgeräte von L. Skohoky (Ungarn), N. Lebedeva (Sowjetunion) und anderen Autoren.

Als Hauptquelle für die Erarbeitung der Kartogramme dienten die ethnographischen Sammlungen der historischen und ethnographischen Museen der baltischen Sowjetrepubliken sowie das in den ethnographischen Abteilungen der historischen Institute der Akademien der Wissenschaften dieser Republiken befindliche Archivmaterial.

Wie die Untersuchung der Flachsbaumethoden der betreffenden Periode im Baltikum zeigt, wurde der Lein auf Brachland als Erstkultur oder auf Sömmerung gesät; beim Dreifeldersystem folgte er auf Roggen und beim Mehrfeldersystem auf zweijährigen,als Untersaat bestellten Klee. Vor der Aussaat hielten die erfahrenen Flachsbauern fleissig nach Federwolken Ausschau, die als günstiges Vorzeichen für eine ergiebige Leinernte galten. Den geeigneten Zeitpunkt für die Aussaat hielt man für gekommen, sobald die Ebereschen blühten. In vielen Fällen bewährten sich diese auf Naturbeobachtung gründenden Regeln: Federwolken am Himmel lassen eine warme und trockene Witterung erwarten, die ein schnelles und gleichmässiges Aufgehen der Leinsaat begünstigt, und die Ebernschen blühen, sobald der Erdboden genügend durchwärmt ist, was ebenfalls eine günstige Voraussetzung für eine erfolgreiche Leinsaat ist.

Bei der Aussaat benutzte man einen Salatkorb oder eine Schürze, die letztere mitunter auch dort, wo bei der Getreidesaat ein Saatkorb verwendet wurde; besonders häufig war das in Litauen der Fall. Die ausgestreuten Samen eggte man ein. Sobald die Saat aufging, wurde gejätet. Allerdings war bis zur Jitte des 19. Jh. das Jäten des Leins nicht allgemein üblich; dieser Arbeitsgang wurde vielfach als entbehrlich betrachtet, und das veranlasste die Agronomen, in ihren Empfehlungen an die Bauern denselben das Jäten der Flachsfelder dringend nahezulegen. ${ }^{1}$ Nur der Baron von Meyendorff, der auf seinem Besitztum Kleinroop den Flachs nach flämischer Methode aufbereiten liess, achtete streng darauf, dass die Fel-

${ }^{1}$ Der Flachsbau. - KLM, 1851, Nr. 14. 
der gejätet wurden. Auch im Kreise Wilkomir (Litauen) war das Jäten der Leinpflanzungen üblich. ${ }^{1}$

Der Ende Mai gesäte Flachs wurde in der ersten Augusthälfte gerauft. Die Bauern hielten die Erntezeit für gekommen, sobald sich die Stengel gelblich und die Samenkapseln bräunlich färbten und die unteren Blätter vertrockneten. Das Raufen wurde von den Frauen besorgt. Sie bündelten den Lein und stellten je zehn oder mehr Leinbündel zu kleinen Schobern zusammen.

Die weitere Flachsaufbereitung erfolgte in verschiedener Weise, je nachdem man den Lein weichte (röstete) oder ihn ungeweicht zum Trocknen auslegte (Karte 1). ${ }^{2}$

Wenn der Lein nicht geröstet wurde, liess man die Bündel bei gutem Wetter auf freiem Feld und anschliessend in der Korndarre trocknen. Danach hieb man den Bündeln mit einem Schlegel die Samenkapseln ab und legte die Flachsstengel auf einer Wiese oder einem Stoppelfeld zum Bleichen aus. Wurde der Lein geweicht, so schlugen die Männer die Samenkapseln gleich nach dem Raufen ab. Gegen Ende des 18. Jh. wurden in den Gouvernements Estland und Livland die Samenköpfchen auf einer Sensenklinge oder mit einem Beil abgeschlagen. Pastor A. Hupel, der Ende des 18. Jh. die aus den Gemeinden Livlands und Estlands eingegangenen Antworten auf eine Rundfrage der Gemeinnützigen ökonomischen Gesellschaft Livlands auswertete, bemerkt hierzu: "Nur auf wenigen IIöfen ist der sogenannte Flachsreffel (eine Art von grober Heckel oder eisernem Kamm) bekannt; man schneidet sie mit einer Sense oder haut sie mit einem Beil ab, wodurch der Flachs von seiner Länge verliert und auch ein Teil des Samens verloren gehet."3

Seit den 20er Jahren des 19. Jh. war der Export von Faserpflanzen über den Rigaer Hafen in stetigem Steigen begriffen. Innerhalb von 70 Jahren (1783-1852) erhöhte sich die Flachs-

1 KLM, 1852, Nr. 16.

Die in diesem Bericht veröffentlichten Karten sind Arbeitskarten, die im weiteren Verlauf der Forschung noch präzisiert werden.

${ }^{3}$ A. Hupel, Topographische Nachrichten von Lief- und Ehstland, II. Riga, 1777 , S. 270. 


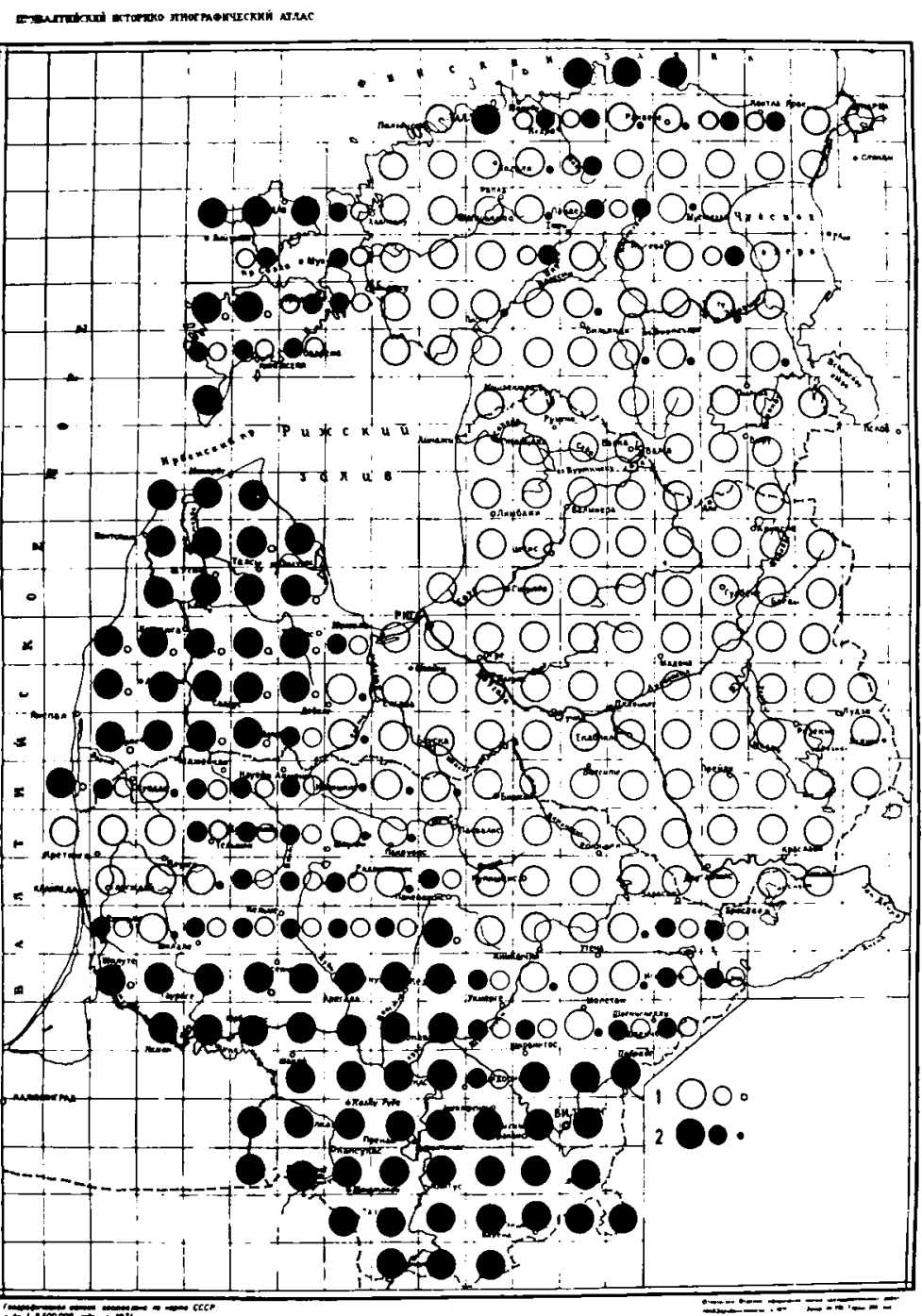

Karte 1. Die Verbreitung der Anwendung von Taurösten und Wasserrösten: 1 - Wasserröste; 2 - Tauröste. (Autoren: V. Kalits, I. Leinasare, A. Vischnewskaite) 
ausfuhr auf das 3,5-fache. ${ }^{1}$ Dies war eine Folge der erhöhten Nachfrage auf den Märkten Europas im Zusammenhang mit der Entwicklung der maschinellen Flachsspinnerei, die zu einer Vergrösserung der Leinanbauflächen führte. In Est-, Liv- und Kurland wirkte sich auch die Aufhebung der Leibeigenschaft und der Übergang zur Geldpacht günstig auf die Entwicklung des Flachsbaus aus. Für den Export benötigte man sorgfältig aufbereiteten, hellen und langfaserigen Flachs, und dieser Umstand führte zu gewissen Verbesserungen und Neuerungen in der Aufbereitungstechnik. Die Benutzung des Flachskammes breitete sich aus, und nur noch die äussersten Enden der Bündel wurden an einer Sensenklinge abgeschlagen. Im Baltikum gab es zwei unterschiedliche Verfahren zum Durchkämmen der Flachsbündel. Das eine bestand darin, dass man den Kamm an einem Pfahl oder einer Bank befestigte und das Flachsbündel durch den Kamm zog. Dieses Verfahren war in Livland, im östlichen Teil Kurlands sowie in den zentralen und südlichen Gebieten Estlands und Litauens verbreitet. Das andere Verfahren bestand darin, dass man mehrere Flachsbündel an einer Bank befestigte und sie durchkämmte. Diese Methode war vorwiegend im östlichen Teil des Baltikums, in Westlitauen und im äussersten Südwesten Lettlands üblich, wie auch bei den russischen und weissrussischen Bauern. Ursprünglich war sie auch im nordöstlichen Teil Litauens verbreitet, doch ging man dort seit dem Ende des 19. Jh. allmählich zum erstgenannten Verfahren über, bei dem der Flachskamm an einem Pfahl befestigt wurde (Karte 2 und Abb. 1).

Nachdem der Flachs von den Samenkapseln befreit war, weichte man ihn in einer Wassergrube (märks) ein, um die Fasern vom Holzstoff zu lösen. Hierzu bemerkt Pastor Hupel: "In der Wahl des Wassers zum Einweichen oder Rösten sind wir nicht sehr vorsichtig: das nächste das beste. Im stehenden Morastwasser wird er etwas schwarz oder gelb, aber zärter; im Flusswasser weisser, aber härter von Haaren."'2 In den $40 \mathrm{er}$ bis 50er Jahren des 19. Jh. begannen einige Flachsbau treibende

1 J. Zutis, a.a.O., S. 22.

2 A. Hupel, a.a.O., S. 270. 


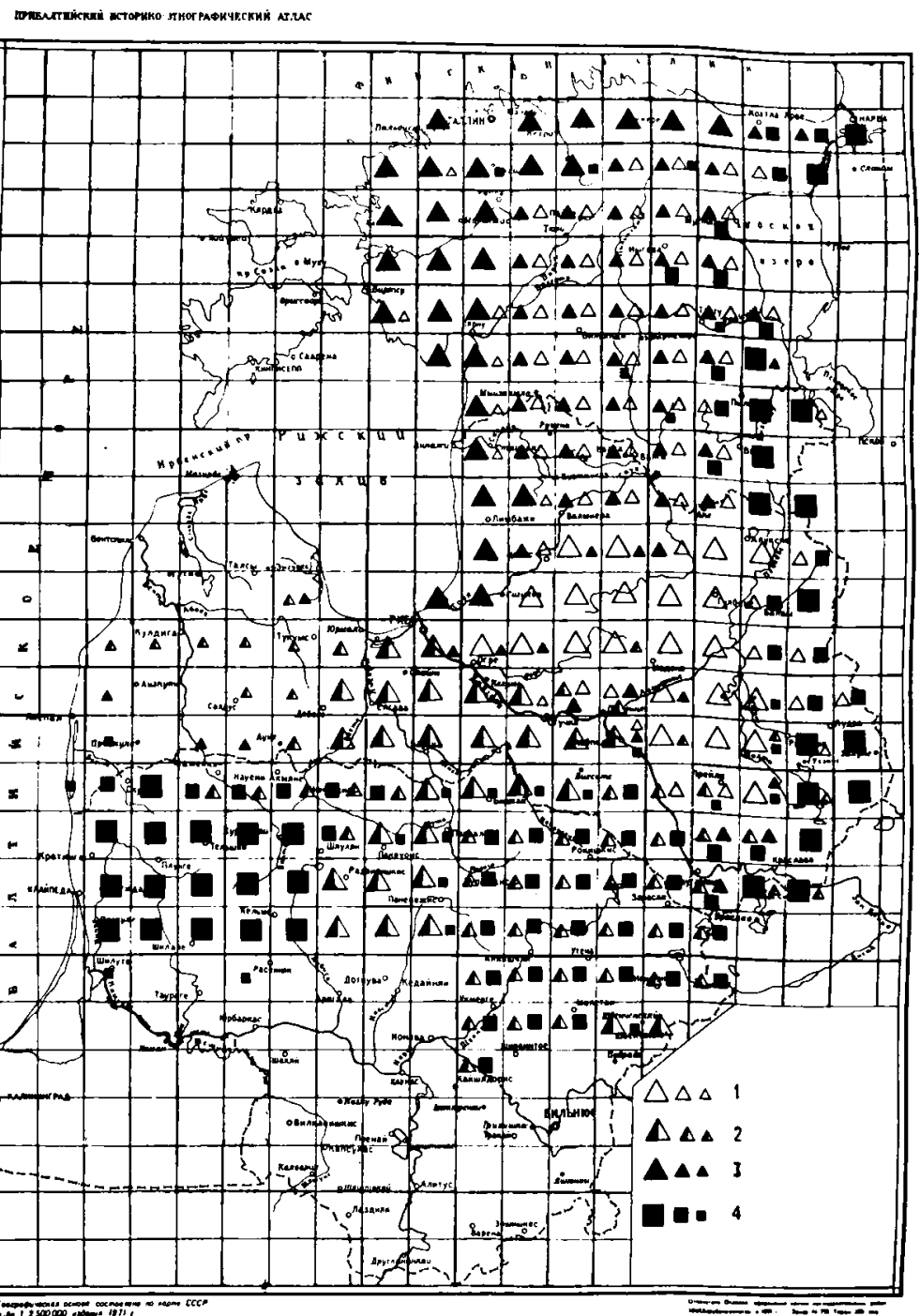

Karte 2. Riffeltypen für das Flachsriffeln:

1. Das Riffelbrett ist an einem pyramidenförmigen Bock befestigt.

2. Das Riffelbrett ist an einem Pfahl befestigt.

3. Die Riffelbank mit dem Riffelbrett.

4. Die Bank, in der das Flachsbündel befestigt und durchgekämmt wird.

(Autoren: V. Kalits, I. Leinasare, A. Vischnewskaite) 

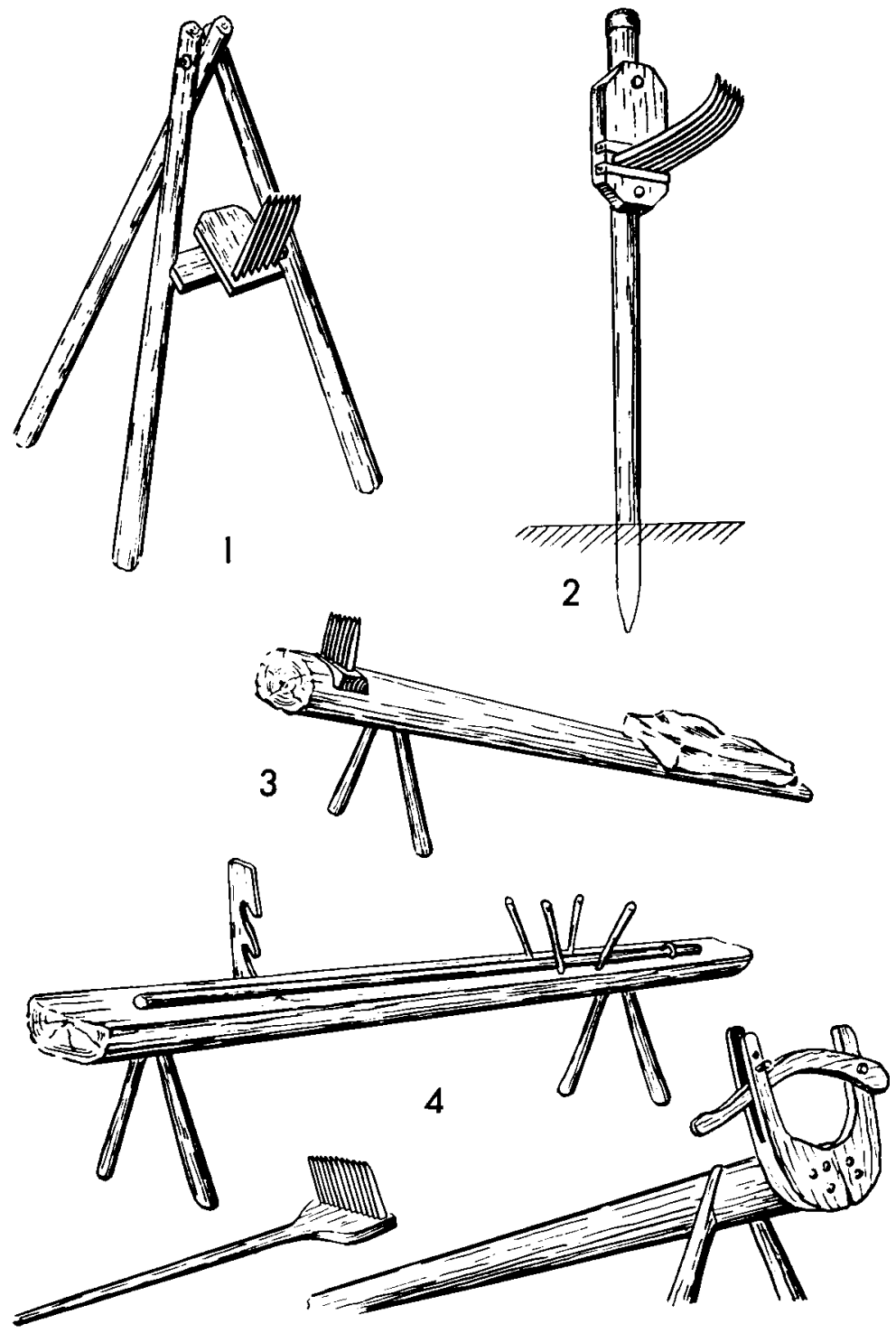

Abb. 1. - Zur Karte 2. 
Gutsbesitzer, um die Qualität ihrer Erzeugnisse bemüht, in den liv- und kurländischen landwirtschaftlichen Zeitschriften die in Irland und Belgien gebräuchlichen Flachsaufbereitungsmethoden zu propagieren. Dem Beispiel dieser Länder folgend, suchten die kurländischen Gutsbesitzer, um hochwertigen Flachs zu gewinnen, das Rösten auf ihren Gütern einzubürgern. Wenn dies in Kurland auch nicht allgemeine Verbreitung fand, begann dennoch ein Teil der Bauern, den Flachs zu rösten. Seit der Mitte und der zweiten Hälfte des 19. Jh. schenkte man auch der Qualität des Röstwassers grössere Beachtung. Um die darin enthaltenen Eisensalze abzusondern, durch die graue, durch Bleichen nicht zu beseitigende Flecken an den Fasern verursacht wurden, weichten die Bauern bereits im Sommer Wacholdersträuche und junge Schwarzerlen in den Wassergruben ein. In dunkle, trübe Wassergruben schüttete man Asche, um ihren Säuregehalt herabzusetzen.

Der Flachs wurde in Estland, im mittleren und östlichen Teil Lettlands sowie in Nordlitauen geweicht, während man ihn in Mittel- und Südlitauen, im westlichen Teil Lettlands (Kurland) und auf den estnischen Inseln ungeröstet auf einer Wiese auslegte. Die ungeweichte Leinfaser war brüchiger und dunkler als die geröstete und nicht selten fleckig. Die Aufbereitung ohne Rösten war hauptsächlich in denjenigen Bauernwirtschaften üblich, die sich auf die Produktion von I einsamen spezialisierten.

Auch der geröstete Flachs wurde auf freiem Felde gebleicht. Zu diesem Zweck breitete man ihn ungebündelt auf einer Wiese oder einem Stoppelfeld aus. Nur im westlichen und südlichen Teil Estlands sowie in Nordlivland wurde er bündelweise auf dem Felde niedergelegt.

Das Rösten des Flachses wurde von den Männern, das Bleichen von den Frauen besorgt.

Im Winter, wenn der Getreidedrusch beendet war, begann das Brechen und Schwingen des Flachses. Um die Leinstengel brüchiger zu machen, dörrte man sie in der Korndarre, mancherorts auch in der Sauna oder, wie in Südwestlitauen, in einer eigens dazu bestimmtem grubenförmigen Flachsdarre.

Derartige Darren wurden in den südwestlichen Gebieten 
Litauens meist an einem Abhang eingerichtet. Dazu hob man eine $3,5 \mathrm{~m}$ lange, $2,5 \mathrm{~m}$ breite und $2 \mathrm{~m}$ tiefe Grube aus, die durch einen Gang mit einer Feuerstelle verbunden war. Die Grube wurde mit Holzstangen bedeckt, auf die man die Flachsbündel zum Dörren legte. Auf diese Weise konnten gleichzeitig 14 Bündel gedörrt werden. Dort am Grubenrand wurde der Flachs auch gebrochen.

Das nächste Kartogramm ist den Geräten zum Brechen des Flachses gewidmet. Gegen Ende des 19. Jh. gab es im Baltikum zwei Typen von Flachsbrechen: zylinderförmige und aus Brettern gefertigte (Abb. 2). Die ersten bestanden in der Hauptsache aus einem Baumklotz, auf dem in Längsrichtung mehrere Schneiden bildende Rinnen eingeschnitten waren; darüber befand sich ein Deckel mit zwei ebensolchen Schneiden, die in die Rinnen des Klotzes eingriffen. Eine solche Flachsbreche hatte meist nur vorn zwei Füsse, während das hintere Ende entweder auf dem Boden lag oder sich auf einen Bock, einen Schlitten oder einen Holzklotz stützte. Die aus Brettern gefertigten Flachsbrechen hatten gewöhnlich vier Füsse.

Der erstgenannte Typus, der in Osteuropa sehr verbreitet war, scheint älteren Ursprungs zu sein; der ungarische Ethnograph Skolnoky datiert seine Entstehung auf das 15. bis 16 . Jahrhundert. Der zweite Typus kam später auf und war vorwiegend im südlichen und mittleren Teil Litauens sowie in manchen Gegenden Westlettlands verbreitet. Im Baltikum begnügte man sich (im Gegensatz zu Finnland) mit nur einer Flachsbreche, an der die Arbeit von Männern verrichtet wurde. Nur im westlichen Teil Litauens wurde ebenso wie in Finnland zur besseren Loslösung der Flachsschäbe noch eine zweite, leichtere Flachsbreche benutzt, die von Frauen bedient wurde.

Die völlige Beseitigung der Schäbe wurde durch das Schwingen des Flaches erreicht; dazu benutzte man verschiedenartig geformte Schwinggeräte. Vom Brechen und Schwingen hing weitgehend die Qualität der Flachsfaser ab. In den 30er und 40er Jahren stellte Baron Meyendorff, um die Rentabilität der Leinpflanzungen zu erhöhen, auf seinem Gut Kleinroop in Mittellivland den Belgier van Steenquist ein, der die Flachs- 

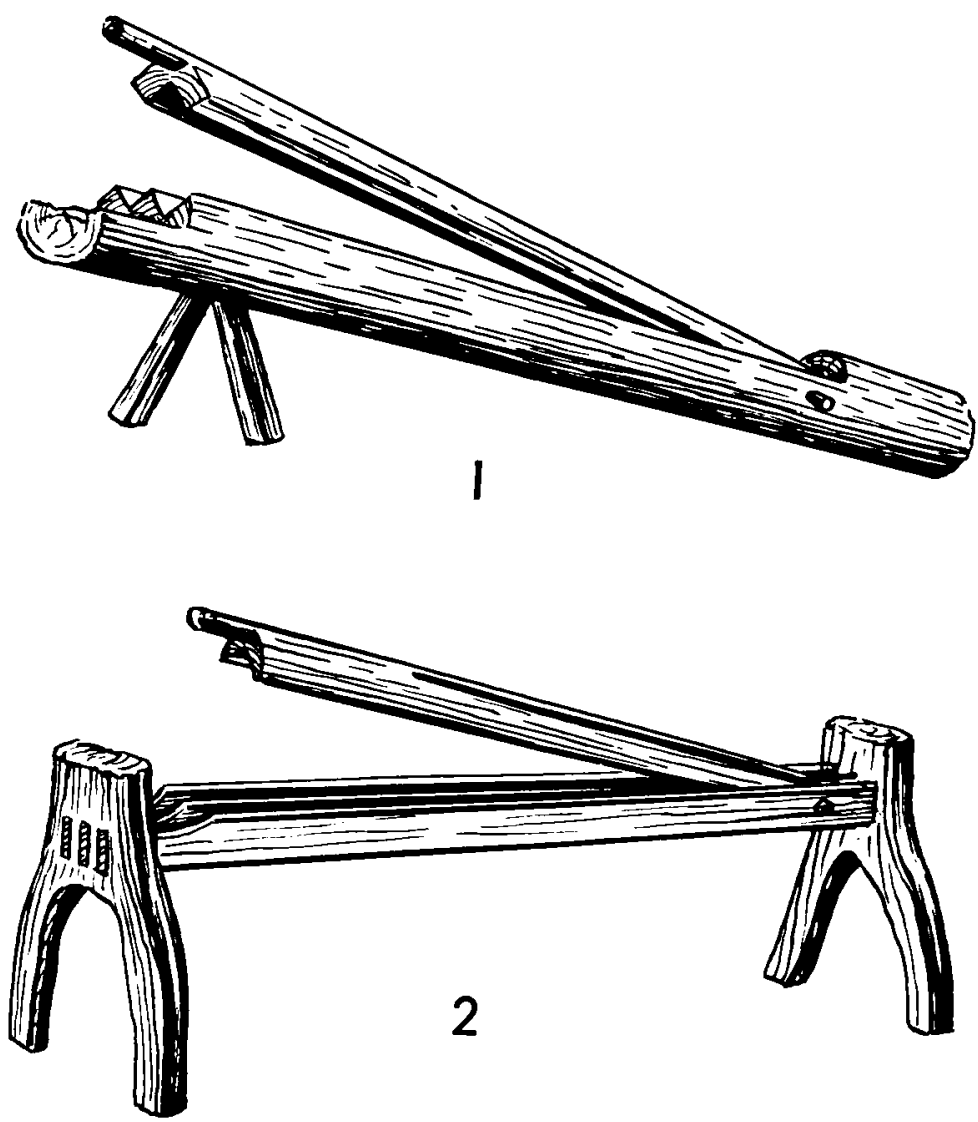

Abb. 2. 1. Die zylinderförmige Flachsbreche.

2. Die aus Brettern gefertigte Flachsbreche.

aufbereitung nach der in Belgien üblichen Methode organisierte. Er führte, um reinere Fasern zu gewinnen, die Bearbeitung mit zwei Flachsschwingen ein und konstruierte eine kleine, mit Pferdekraft betriebene Flachsbrechmaschine. Steenquist vermittelte seine Erfahrungen auch den Bauern, die eigens zu diesem Zweck von den umliegenden Gütern nach Kleinroop geschickt wurden. Imı Jahre 1842 wurde die belgische Flachsaufbereitungsmethode mitsamt den dazu benötigten Maschinen bereits auf mehr als dreissig livländischen Gütern, 
und zwar hauptsächlich in der Umgebung von Gulbene und Verava angewandt. ${ }^{1}$ Während die Empfehlung Steenquists, den Lein in Kästen in fliessendem Wasser zu rösten, bei den Flachsbauern kein besonderes Gehör fand, wurden die von ihm eingeführten Neuerungen beim Schwingen des Flachses bereitwillig übernommen. Am Ende des 19. Jh. arbeitete man bereits im gesamten nördlichen Livland nach der von ihm empfohlenen Methode. Von den wohlhabenden Bauern wurden in der zweiten Hälfte des 19. Jh. auch weitgehend Flachsbrechmaschinen verwendet; auf den Gütern dagegen war ihre Zahl verhältnismässig gering. So besassen beispielsweise im Jahre 1863 nur 10 Prozent aller Güter Flachsbrechmaschinen, während 38,8 Prozent über Dreschmaschinen verfügten. ${ }^{2}$ Es gab Flachsbrechmaschinen von zweierlei Art - Rollen- und Brückenmaschinen. Am verbreitetsten waren die verhältnismässig kleinen und mobilen Rollenmaschinen. Nach der Einbürgerung der Flachsbrechmaschinen benutzte man die alten Flachsbrechen nur noch dazu, um festzustellen, ob die Flachsstengel für die Verarbeitung genügend vorbereitet seien.

Seit Beginn des 20. Jh. wurden in wohlhabenden Bauernwirtschaften auch Schwingmaschinen benutzt.

Wie aus den obigen Ausführungen hervorgeht, gleichen die im Baltikum verwendeten Geräte zur Aufbereitung des Flachses denjenigen Osteuropas.

I. LEINASARE

\footnotetext{
1 Ответ на вопрос об улучшенном пронзводстве льна на мызе Клейн-Рооп. - Земледельческая газета, 1836, Nr. 52.

2 Fr. Jung-Stilling, Material zu einer allgemeinen Statistik Livlands und Ösels. Riga, 1864, S. 216-219.
} 